\title{
Severe osteomyelitis caused by Myceliophthora thermophila after a pitchfork injury
}

\author{
Lauren Destino ${ }^{1}$, Deanna A Sutton ${ }^{2}$, Anna L Helon ${ }^{3}$, Peter L Havens ${ }^{1}$, \\ John G Thometz ${ }^{4}$, Rodney E Willoughby $\mathrm{Jr}^{1}$ and Michael J Chusid*1
}

\author{
Address: ${ }^{1}$ Department of Pediatrics, Medical College of Wisconsin and Children's Hospital of Wisconsin, USA, ${ }^{2}$ Fungus Testing Laboratory, \\ Department of Pathology, University of Texas Health Sciences Center, San Antonio, TX, USA, ${ }^{3}$ Pharmacy Department, Children's Hospital of \\ Wisconsin, USA and ${ }^{4}$ Department of Orthopedic Surgery, Medical College of Wisconsin and Children's Hospital of Wisconsin, USA \\ Email: Lauren Destino - ldestino@mcw.edu; Deanna A Sutton - suttond@UTHSCSA.edu; Anna L Helon - anna.helon@gmail.com; \\ Peter L Havens - phavens@mcw.edu; John G Thometz - jthometz@mcw.edu; Rodney E Willoughby - rewillou@mcw.edu; \\ Michael J Chusid* - mchusid@mcw.edu \\ * Corresponding author
}

Published: 08 September 2006

Annals of Clinical Microbiology and Antimicrobials 2006, 5:2I doi:10.1 I86/1476-07II-5-

21

This article is available from: http://www.ann-clinmicrob.com/content/5/I/2I

(C) 2006 Destino et al; licensee BioMed Central Ltd.

This is an Open Access article distributed under the terms of the Creative Commons Attribution License (http://creativecommons.org/licenses/by/2.0), which permits unrestricted use, distribution, and reproduction in any medium, provided the original work is properly cited.
Received: 26 June 2006

Accepted: 08 September 2006

\begin{abstract}
Background: Traumatic injuries occurring in agricultural settings are often associated with infections caused by unusual organisms. Such agents may be difficult to isolate, identify, and treat effectively.

Case report: A 4-year-old boy developed an extensive infection of his knee and distal femur following a barnyard pitchfork injury. Ultimately the primary infecting agent was determined to be Myceliophthora thermophila, a thermophilic melanized hyphomycete, rarely associated with human infection, found in animal excreta. Because of resistance to standard antifungal agents including amphotericin B and caspofungin, therapy was instituted with a prolonged course of terbinafine and voriconazole. Voriconazole blood levels demonstrated that the patient required a drug dosage $(13.4 \mathrm{mg} / \mathrm{kg}$ ) several fold greater than that recommended for adults in order to attain therapeutic blood levels.
\end{abstract}

Conclusion: Unusual pathogens should be sought following traumatic farm injuries. Pharmacokinetic studies may be of critical importance when utilizing antifungal therapy with agents for which little information exists regarding drug metabolism in children.

Myceliophthora thermophila is a thermophilic phaeoid mould found in pasture soil, wood chips, straw, mouldy hay, compost piles and other environmental settings where heat is generated. It is also found in the excreta and rumen of cattle and is a pathogen of cultivated mushrooms [1]. A rare cause of invasive human infections, it can be difficult to isolate and identify in clinical specimens. We recently cared for a 4-1/2 year old boy who developed osteomyelitis of the distal femur caused by direct inoculation of Myceliophthora thermophila via a pitchfork injury to his knee. The patient demonstrated severe destructive osseous and cartilaginous infection, with slow clinical improvement, requiring the prolonged use of multiple antifungal agents. Due to the limited number of agents to which this organism was susceptible, voriconazole therapy was instituted despite limited phar- 
macokinetic data in children. Prolonged therapy with terbinafine, a drug generally employed for superficial saprophytic infections of skin and nails also was utilized. This case demonstrates the difficulties that can be encountered in identifying and treating this unusual but aggressive fungal organism.

\section{Case report}

A 4-1/2 year old boy presented with a swollen right knee after being impaled in that area by a pitchfork. The pitchfork was observed to be contaminated with cow manure and hay. The knee was washed with soap and water. The following morning the knee was swollen and the boy was treated with orally administered antibiotics.

The mobility of the knee progressively decreased, and four days later the child was admitted to the hospital. Bacterial cultures of joint fluid yielded Bacillus and Enterococcus species. After a brief course of intravenous antibiotic therapy, the boy was discharged to continue orally administered antibiotics. At home, he developed increasing knee pain with inability to walk. A magnetic resonance image (MRI) obtained at transfer to our institution was consistent with infection involving the synovium, the medial femoral condyle and adjacent articular cartilage. Intravenous antibacterial therapy was instituted with vancomycin, piperacillin-tazobactam and amikacin.

Twenty seven days after the initial pitchfork injury, the patient was returned to the operating room because of persistent leg and knee swelling as well as increasing elevation of inflammatory markers with an ESR of $>100$ and a CRP of 6.5. An MRI revealed apparent osteomyelitis of the medial femoral condyle. New bone cultures were obtained which grew what was initially identified as a dermatophyte. Orally administered terbinafine, $125 \mathrm{mg}$ (6.7 $\mathrm{mg} / \mathrm{kg}$ ) daily, was initiated, and the patient began to improve clinically. His CRP declined to a nadir of 1.8 with absence of fever and better movement of his leg. However, 45 days following the initial injury and 14 days after wound closure, an elevation in the CRP to 2.6, as well as an increase of purulent drainage from the knee prompted another surgical exploration of the distal femur and the addition of intravenous Ambisome, $90 \mathrm{mg}(4.8 \mathrm{mg} / \mathrm{kg})$ daily. At surgery, progressive bone loss was noted as well as necrosis of knee cartilage. Fungal organisms with irregular branching hyphae were noted throughout the excised cartilage, and fungus was recovered in culture two weeks later.

Meanwhile, the fungal agent that had been isolated previously was forwarded to the Fungus Testing Laboratory at the University of Texas Health Science Center in San Antonio for identification and susceptibility testing and accessioned into their stock collection as UTHSC 05-3365.
There, the organism was identified as Myceliophthora thermophila, based upon observation of: 1) tan to brown powdery colonies with ill-defined margins when grown on potato flakes agar at $42^{\circ} \mathrm{C} ; 2$ ) more luxuriant growth at elevated temperatures of $35^{\circ} \mathrm{C}$ and $42^{\circ} \mathrm{C}$ than at $26^{\circ} \mathrm{C}$; 3 ) septate vegetative hyphae with conidial production from ampulliform swellings; and 4) obovoid (inverted egg shaped) or pyriform (pear-shaped) conidia measuring $4.5-11.0 \times 3.0-4.5 \mu \mathrm{m}$ that were hyaline and smooth when immature, becoming darker and roughened at maturity (Figure 1). Antifungal susceptibility testing was performed according to the Clinical Laboratory Standards Institute (CLSI) M38-A document for filamentous fungi [2]. Although standardized susceptibility breakpoints have not been established for this organism, the isolate appeared resistant to a variety of standard antifungal agents (Table 1). Based upon these susceptibilities, voriconazole $(4 \mathrm{mg} / \mathrm{kg} / 12 \mathrm{hrs}$ after a loading dose of $6 \mathrm{mg} / \mathrm{kg} /$ 12 hrs for 24 hours) was added to the patient's antifungal regimen, and Ambisome was discontinued. Subsequently, the patient underwent numerous debridement procedures with gradual improvement in clinical picture and CRP. A series of progressive increases in voriconazole dosage was required based upon periodic pharmacokinetic studies to achieve appropriate blood levels (Table 2).

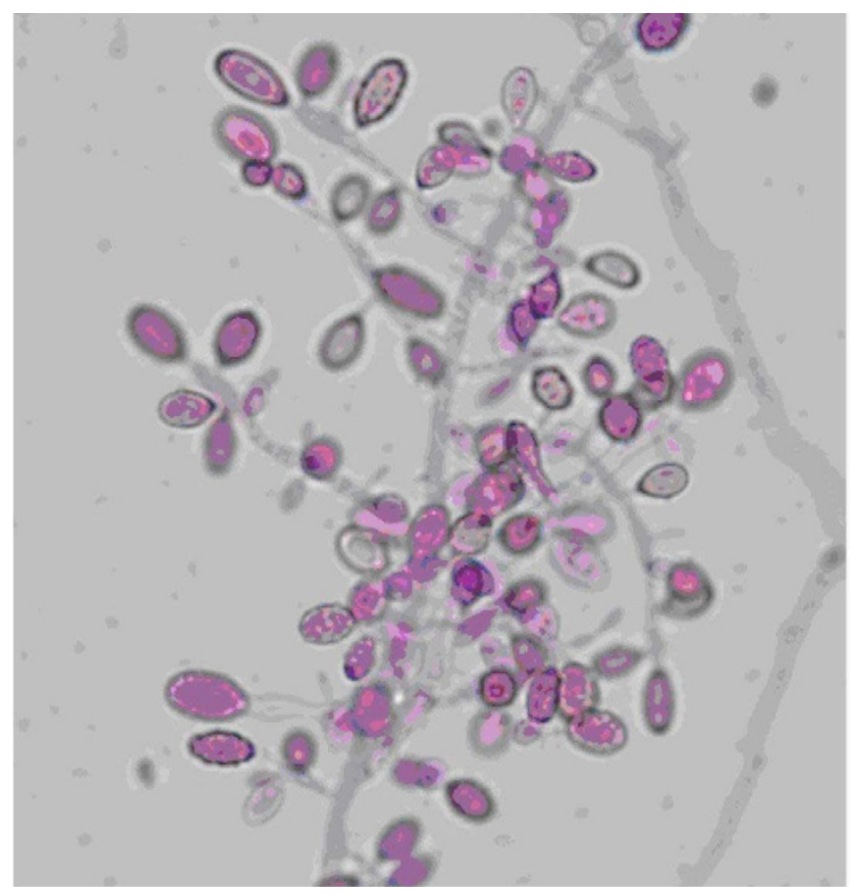

Figure I

Conidia of Myceliophthora thermophila being produced from ampulliform swellings. Note both smooth, hyaline, immature conidia, and darker, more mature roughened conidia. Lactofuschin stain, approximately $1000 \times$. 
Table I: Susceptibilities of Myceliophthora thermophila isolate

\begin{tabular}{ll}
\hline Drug & MIC \\
\hline Amphotericin B & 2 resistant* \\
Fluconazole & 8 resistant \\
Itraconazole & 0.125 susceptible \\
Voriconazole & 0.06 susceptible \\
Caspofungin & 4 resistant \\
Terbinafine & 1 susceptible \\
Griseofluvin & $>16$ resistant
\end{tabular}

*There are no published breakpoints for this organism against any of the antifungal agents tested. Interpretations are based upon normally achievable concentrations of the drug using standard dosing regimens.

After his CRP had normalized, the patient was discharged home receiving voriconazole, $250 \mathrm{mg}$ (13.4 mg/kg) orally, every 12 hours, and terbinafine $125 \mathrm{mg}(6.7 \mathrm{mg} /$ $\mathrm{kg}$ ) orally once daily. Anti-fungal therapy is to be continued for a total of one year. The leg wound has healed, but the patient has had significant bone loss in his distal femur with involvement of the growth plate, as well as damage to the articular cartilage of the knee.

\section{Discussion}

Myceliophthora thermophila is a melanized filamentous hyphomycete that initially grows as a white cottony colony and subsequently turns pale brown and becomes granular on a variety of media recommended for mould identification, such as potato dextrose or $2 \%$ malt agar. Its optimal growth is at $30-36^{\circ} \mathrm{C}$. However, it also grows well at $42^{\circ} \mathrm{C}$, with maximal growth near $50^{\circ} \mathrm{C}$. Thus it is considered a thermophilic organism. Myceliophthora thermophila is found in dry pasture soil, birch chips, wood pulp, and straw compost [1]. Its cell wall contains melanin resulting in dark pigmentation, and it is considered one of the etiologic agents of phaeohyphomycosis. Phaeohyphomycosis includes those conditions in which the pathogenic mould forms fungal elements which contain melanin within their cell walls [3]. Despite the presence of melanin, cell walls of phaeoid moulds may appear hyaline or clear upon routine microscopy. Hyphal ele- ments usually demonstrate pigment when stained with Masson-Fontana melanin stain, allowing identification of a dark fungus [4].

A recent review reports that the number of publications related to phaeohyphomycotic infections in the 1990's numbered only 150 [5]. Phaeohyphomycosis most commonly manifests as a cutaneous infection, but deep infections with invasion of the sinuses, lungs, brain, blood, and bone have also been reported [5]. Disseminated disease was reviewed by Revankar et al. who found 72 cases reported between 1966 and 2001 [6]. Notably, the majority of cases involving disseminated phaeohyphomycosis were in immune-compromised patients. The mortality rate in these individuals was high and many isolates were resistant to amphotericin B. In immunocompetent patients, most infections were associated with direct inoculation of the organism from an environmentally contaminated source.

There are just three previously reported cases of phaeohyphomycosis caused by Myceliophthora thermophila (Table 3). In the first two patients, the source of the Myceliophthora thermophila was uncertain, and both patients died despite standard anti-fungal therapy with amphotericin $\mathrm{B}$. The most recently reported case of infection with this organism involved a 21-month-old boy who sustained a penetrating head injury. A brain abscess developed from which both Clostridium perfringens and Myceliophthora thermophila were isolated. The patient was treated successfully with enbloc resection of the lesion, six weeks of amphotericin B, and four months of itraconazole [9].

Despite convincing evidence of progressive infection in each of the three previously reported cases, it was not until well into the clinical course or even after death that identification of the etiologic agent was confirmed. It is unknown why recovery of Myceliophthora thermophila from clinical specimens is so difficult. However, the situation may be analogous to mycotic infections with more

Table 2: Voriconazole plasma concentrations (body weight 18.6 kg)*

\begin{tabular}{clcccc}
\hline Voriconazole Therapy Day & Dose $(\mathrm{mg})$ given every I2 hr & Dose $(\mathrm{mg} / \mathrm{kg})$ & Doses prior to kinetics & Peak $(\mathrm{mcg} / \mathrm{ml})$ & Trough $(\mathrm{mcg} / \mathrm{ml})$ \\
\hline 6 & 75 IV & 4 & 10 & $0.94^{\mathrm{a}}$ & $<0.2$ \\
I4 & I 08 IV & 5.8 & 8 & 0.6 & $<0.2$ \\
24 & I 75 IV & 9.4 & 6 & 3.04 & $2.8^{\mathrm{b}}$ \\
34 & 250 PO & 13.4 & 8 & 5.35 & 0.3 \\
43 & 250 PO & 13.4 & 54 & $2.12^{\mathrm{b}}$ & 0.3 \\
57 & 250 PO & 13.4 & 54 & 0.2 \\
\hline
\end{tabular}

\footnotetext{
*Patient also receiving terbinafine $6.7 \mathrm{mg} / \mathrm{kg} /$ day

alV peak@ 050 minutes post infusion

bPO peak@2-3 hours post ingestion

c PO peak @ <2 hours post ingestion
} 
Table 3: Prior Case Reports of Myceliophthora thermophila infection

\begin{tabular}{|c|c|c|c|c|}
\hline Age, Sex & History & Sites of Infection & Therapy & Outcome \\
\hline 7 years, $M^{7}$ & AML with neutropenia & Blood, lungs, heart & Amphotericin B & Death \\
\hline 22 years, $\mathrm{F}^{8}$ & Status post Cardiovascular Surgery & Blood, aorta, heart & Amphotericin B 5-fluorocytosine & Death \\
\hline 21 months, $M^{9}$ & 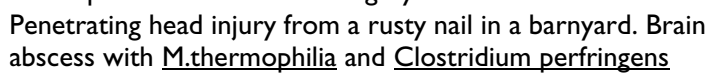 & Brain abscess & Amphotericin B Itraconazole & Survived \\
\hline
\end{tabular}

common agents such as Aspergillus, in which microbiologic isolation of the etiologic agent from grossly infected tissue can be difficult. The identification of Myceliophthorathermophila, once recovered, is also problematic, as most microbiology laboratories lack experience with this organism.

In our patient, despite evidence of ongoing infection, both operatively and preoperatively, only two samples of debrided bone or cartilage yielded Myceliophthora thermophila, despite numerous cultures of infected surgical specimens in which fungal elements could be seen histologically. Given the thermophilic nature of this organism, incubation of inoculated media at elevated temperatures may enhance recovery. Cultures of most clinical specimens are incubated at $30^{\circ} \mathrm{C}$, but such a temperature would be less than optimal for growth of Myceliophthora thermophila.

Because our patient's positive cultures was obtained after approximately 2 weeks of terbinafine therapy and the organism was found to be resistant to amphotericin B, voriconazole was added to terbinafine therapy. Terbinafine is a broad-spectrum allylamine with fungicidal activity against dermatophyte species, Aspergillus species, Sporothrix schenckii, Blastomyces dermatitidis, Histoplasma capsulatum, Cryptococcus neoformans, Malassezia furfur and other important fungi. It shows in vitro synergism with amphotericin or triazoles and has been effective in combination therapy in individual patients [10-14]. It has been administered safely in a large number of children, and at high doses or for up to 12 months for invasive mycoses [15].

Voriconazole is a potent antifungal agent effective against a number of pathogens, including Aspergillus, Cryptococcus, and Candida species. It also has excellent oral bioavailability and a low rate of adverse effects $[16,17]$. However, it is not approved by the Food and Drug Administration for use in children, and the appropriate dose for pediatric patients is not known. Recommendations in authoritative sources suggest the same intravenous weight-based dosages in children and adults: a loading dose of $6 \mathrm{mg} / \mathrm{kg} /$ dose every 12 hours $\times 1$ day and a maintenance dose of $4 \mathrm{mg} / \mathrm{kg} /$ dose every 12 hours. Oral dosage is suggested at $100 \mathrm{mg}$ every 12 hours for patients less than $40 \mathrm{~kg}$, and $200 \mathrm{mg}$ every 12 hours for patients more than $40 \mathrm{~kg}$ [18].

Recent investigations by Walsh et al. demonstrated that pediatric patients have a much higher rate of elimination of voriconazole per unit of body weight than do adults. Thus, children may require higher dosages to achieve blood levels consistent with adults treated at a dosage of $3-4 \mathrm{mg} / \mathrm{kg}[16,17]$. Additionally, the elimination of voriconazole from the blood in children appears linear when doses of $3 \mathrm{mg} / \mathrm{kg}$ to $5 \mathrm{mg} / \mathrm{kg}$ are administered every 12 hours. This is in distinction to elimination of similar doses in adults, which is non-linear or saturable. Although the exact relationship between the plasma concentration of voriconazole and the drug's clinical effectiveness is uncertain, infected adults improve at doses achieving recommended plasma concentrations. Therefore, the goal in our patient was to achieve voriconazole blood levels similar to those achieved in adults. Assuming linear pharmacokinetics, it was suggested by Walsh that a pediatric dosage of up to $11 \mathrm{mg} / \mathrm{kg}$ twice a day might be necessary to achieve drug levels equivalent to those seen in adult patients receiving $4 \mathrm{mg} / \mathrm{kg}$ of the agent every 12 hours [17]. We increased the dose of voriconazole in our patient, in stepwise fashion based upon measurements of $\mathrm{C}_{\text {max }}$, from $4 \mathrm{mg} / \mathrm{kg}$ to $13.4 \mathrm{mg} / \mathrm{kg}$ every 12 hours, reaching a voriconazole serum peak levels in the range of 2-5 $\mathrm{mcg} / \mathrm{ml}$, equivalent to the typical adult given only 3-4 $\mathrm{mg} / \mathrm{kg}$ every 12 hours. Kinetics of voriconazole in our patient were potentially affected by concurrent administration of terbinafine.

The current case demonstrates the vigilance required in patients with traumatically induced osteomyelitis, particularly when related to direct implantation from a grossly contaminated source. Relapse of apparently appropriately treated infection while on therapy demands reassessment to be certain that an unusual or emergent microorganism is not present within the depths of the wound. In the case of a contaminated farm implement, the possibility of recovery of an unusual agent like Myceliophthora thermophila is high, potentially requiring the usage of antimicrobial agents for which there is scant pharmacologic data in children. Clinicians should be careful to obtain appropriate pharmacologic studies in order to be assured that the appropriate doses of such drugs are employed. 


\section{Authors' contributions}

LD conceived the report and helped in its writing. DS oversaw antimicrobial susceptibility testing and voriconazole blood level determinations. AH performed voriconazole pharmacokinetic analysis. $\mathrm{PH}$ provided clinical care and editorial assistance. JT provided surgical care and the tissue specimens from which the pathogen was recovered. RW provided clinical care and conceived the antimicrobial regimen for this patient. MC provided clinical care, editorial support and helped conceive this paper.

\section{Acknowledgements}

The authors would like to acknowledge Elizabeth Thompson and Gen Pennick of the Fungus Testing Laboratory for their work in the identification of the isolate and performance of voriconazole levels, respectively. The patient's parents agreed in writing to the publication of this report.

\section{References}

I. CAN O: The genus Myceliophthora. Persoonia 1977, 9:40I-408.

2. National Committee for Clinical Laboratory Standards: Reference method for broth dilution antifungal susceptibility testing of filamentous fungi: approved standard. Volume NCCLS document M38-A. Wayne, PA, Clinical Laboratory Standards Institute; 2002.

3. Ajello L: Hyalohyphomycosis and phaeohyphomycosis: two global disease entities of public health importance. Eur J Epidemiol 1986, 2:243-25I.

4. Rinaldi MG: Phaeohyphomycosis. Dermatol Clin 1996, I4: I47-I53.

5. Silveira F, Nucci M: Emergence of black moulds in fungal disease: epidemiology and therapy. Curr Opin Infect Dis 200I, 14:679-684.

6. Revankar SG, Patterson JE, Sutton DA, Pullen R, Rinaldi MG: Disseminated phaeohyphomycosis: review of an emerging mycosis. Clin Infect Dis 2002, 34:467-476.

7. Bourbeau P, McGough DA, Fraser H, Shah N, Rinaldi MG: Fatal disseminated infection caused by Myceliophthora thermophila, a new agent of mycosis: case history and laboratory characteristics. J Clin Microbiol 1992, 30:3019-3023.

8. Farina C, Gamba A, Tambini R, Beguin H, Trouillet JL: Fatal aortic Myceliophthora thermophila infection in a patient affected by cystic medial necrosis. Med Mycol 1998, 36: I I3-I I8.

9. Tekkok IH, Higgins MJ, Ventureyra EC: Posttraumatic gas-containing brain abscess caused by Clostridium perfringens with unique simultaneous fungal suppuration by Myceliophthora thermophila: case report. Neurosurgery 1996, 39:|247-|25|.

10. Gosbell IB, Toumasatos V, Yong J, Kuo RS, Ellis DH, Perrie RC: Cure of orthopaedic infection with Scedosporium prolificans, using voriconazole plus terbinafine, without the need for radical surgery. Mycoses 2003, 46:233-236.

II. Gupta AK, Taborda PR, Sanzovo AD: Alternate week and combination itraconazole and terbinafine therapy for chromoblastomycosis caused by Fonsecaea pedrosoi in Brazil. Med Mycol 2002, 40:529-534

12. Howden BP, Slavin MA, Schwarer AP, Mijch AM: Successful control of disseminated Scedosporium prolificans infection with a combination of voriconazole and terbinafine. Eur / Clin Microbiol Infect Dis 2003, 22: III-113.

13. Rothe A, Seibold M, Hoppe T, Seifert H, Engert A, Caspar C, Karthaus M, Fatkenheuer G, Bethe U, Tintelnot K, Cornely OA: Combination therapy of disseminated Fusarium oxysporum infection with terbinafine and amphotericin B. Ann Hematol 2004, 83:394-397.

14. Ryder NS, Leitner I: Synergistic interaction of terbinafine with triazoles or amphotericin B against Aspergillus species. Med Mycol 200I, 39:91-95.

15. Gupta AK, Adamiak A, Cooper EA: The efficacy and safety of terbinafine in children. I Eur Acad Dermatol Venereol 2003, 17:627-640.

16. Walsh TJ, Lutsar I, Driscoll T, Dupont B, Roden M, Ghahramani P, Hodges M, Groll AH, Perfect JR: Voriconazole in the treatment of aspergillosis, scedosporiosis and other invasive fungal infections in children. Pediatr Infect Dis J 2002, 21 1:240-248.

17. Walsh TJ, Karlsson MO, Driscoll T, Arguedas AG, Adamson P, SaezLlorens X, Vora A], Arrieta AC, Blumer J, Lutsar I, Milligan P, Wood $\mathrm{N}$ : Pharmacokinetics and safety of intravenous voriconazole in children after single- or multiple-dose administration. Antimicrob Agents Chemother 2004, 48:2166-2I72.

18. Taketomo C, Hodding J, Kraus D: Pediatric Dosage Handbook I2th edition. Hudson, Ohio, Lexi-Comp; 2006.
Publish with Bio Med Central and every scientist can read your work free of charge

"BioMed Central will be the most significant development for disseminating the results of biomedical research in our lifetime. "

Sir Paul Nurse, Cancer Research UK

Your research papers will be:

- available free of charge to the entire biomedical community

- peer reviewed and published immediately upon acceptance

- cited in PubMed and archived on PubMed Central

- yours - you keep the copyright 